\title{
Recombinant Fas Ligand
}

National Cancer Institute

\section{Source}

National Cancer Institute. Recombinant Fas Ligand. NCI Thesaurus. Code C1538.

A recombinant agent, which is chemically identical to or similar to the endogenous protein Fas lig and, a protein related to tumor necrosis factor (TNF) with potential antineoplastic activity. Fas ligand binds to the Fas receptor, thereby activating caspases and inducing apoptosis. ( $\mathrm{NCIO4)}$ 